\title{
On the natural frequencies and mode shapes of dragonfly wings
}

\author{
Jen-San Chen*, Jeng-Yu Chen, Yuan-Fang Chou \\ Department of Mechanical Engineering, National Taiwan University, Taipei 10617, Taiwan
}

Received 17 February 2007; received in revised form 23 July 2007; accepted 30 November 2007

Available online 4 January 2008

\begin{abstract}
A base-excitation modal testing technique is adopted to measure the natural frequencies and mode shapes of dragonfly wings severed from thoraxes. The severed wings are glued onto the base of a shaker, which is capable of inducing translational motion in the lateral direction of the wing plane. Photonic probes are used to measure the displacement history of the shaker base and the painted spots of the wing simultaneously. A spectrum analyzer is employed to calculate the frequency response functions, from which the natural frequencies and the associated mode shapes of the wing structure can be extracted. Our experimental results show that the fundamental natural frequency of dragonfly wings is in the order of $170 \mathrm{~Hz}$ when it is clamped at the wing base. The average flapping frequency $27 \mathrm{~Hz}$ of dragonflies is about $16 \%$ of the fundamental natural frequency. At this frequency ratio, the inertial force of the wing is negligible compared to the elastic force. In other words, the wing deformation during flapping flight is solely due to the balance between the external aerodynamic force and the elastic force of the wing structure. The wing structures are generally lightly damped, with damping ratio in the order less than $5 \%$.
\end{abstract}

(C) 2007 Elsevier Ltd. All rights reserved.

\section{Introduction}

Many of the previous theories in calculating aerodynamic forces produced during insect flight are based on the assumption that the insect wings are rigid. However, it has been observed that many insect wings undergo significant bending and twisting deformations during flight [1]. How to incorporate the wing flexibility into the theoretical model predicting the aerodynamic force during insect flight remains an ongoing challenge to the researchers.

Insect wings are mainly passive structures. In other words, the insects have no active control over the wing configuration during flight. The architecture of the wing and the material properties of its element determine how the wing changes shape in response to external forces. Recently, the measurement of the mechanical properties of insect wings has attracted research attention because it may provide useful information in establishing mathematical models in predicting the aerodynamic force during insect flight. The first step in this research direction is to measure the stiffness of the insect wings. Newman and Wootton [2] applied point force to isolated wing section to measure the stiffness of dragonfly wings. Ennos [3] investigated the torsional deformation of fly wings. Wootton [4] conducted torsional tests on the forewings of butterflies. Combes and

*Corresponding author. Tel.: + 886223661734 ; fax: + 886223631755 .

E-mail address: jschen@ntu.edu.tw (J.-S. Chen). 
Daniel [5] performed bending tests on 16 insect species from six orders in an effort to clarify the functional significance of phylogenetic trends in wing venation. These measurements can be classified as static tests as they quantify the ability of the wing structures in resisting deformation when the inertial force is neglected.

The static tests on the insect wings mentioned above offer us a glimpse on the order of magnitude of the insect wing stiffness. However, during insect flight the wings are under periodic excitation instead of static force. As a consequence, the wings are subject to inertial force resulting from the acceleration of the wing mass, elastic force tending to resist any wing deformation, external aerodynamic force responsible for the lift and thrust of the insect body, and the force exerted by flight muscle at the wing base. Recently, there have been some debates on the role of inertial force on the wing deformation during insect flight. It was conjectured [6] that the contribution of the aerodynamic force on the wing deformation is minor compared to the inertial and elastic forces. In other words, the wing deformation is mainly the result of the force balance between inertial force and elastic force. If this is true, it means that the wings are vibrating near their natural frequency [7] when the insect flaps its wings. In this paper, we examine this conjecture by conducting a modal testing on the wings to determine the natural frequencies, the mode shapes, and the damping ratio of the wing structures.

One of the challenges facing modern modal testing technique is to measure the dynamic characteristics of miniature structures. In hard-disk drives industry, various techniques have been proposed to measure the dynamic parameters of the tiny read/write head suspension. Radwan and Chokshi [8] fixed the suspension at one end and excited it by a mechanical shaker at the other end. A small force transducer is used to measure the force applied by the shaker, and a photonic probe is used to measure the response. Castagna [9] utilized the suspension's servo actuator as the excitation device and used the white noise current in the servo actuator as the force portion of the frequency response function. Patton and Trethewey [10] used electromagnetic exciter to perform modal testing on miniature structures. The electromagnetic actuator focused on a small ferromagnetic target, which is glued onto the test structure. These electromagnetic exciters are designed to provide a force which approximates a point load. Wilson and Bogy [11] refined the design of the electromagnetic exciter and improved the technique of sputtering ferromagnetic target on the structure. Miu et al. [12] used a pulse air to induce free vibration of a head-suspension assembly, and used a laser Doppler vibrometer to measure its response.

One of the difficulties encountered in conventional modal testing as mentioned above lies in the fact that no reliable device is capable of exerting and measuring excitation force at precise location of the structure. However, this problem can be substantially eliminated by the base-excitation method. The structure under testing is attached to a rigid base that is driven by a mechanical shaker. The input measurement used in calculating the frequency response function is the displacement of the base, and the output measurement is the displacement at various points on the flexible structure. In order to utilize currently available modal analysis software, Beliveau et al. [13] outlined a procedure to modify the frequency response function for obtaining modal information of the structure. Henze et al. [14] applied this method to measure the modal parameters of a type-4 suspension assembly. Although the base-excitation technique appears to give satisfactory result when the structure under testing is small, its theoretical formulation was based on the assumption of a discrete model. Since all real structures are continuous, it is desirable to formulate the modal analysis technique within the framework of a continuous system model. In this paper, we formulate the base-excitation modal testing technique within the framework of a continuous system and apply the method to extract the dynamic characteristics of dragonfly wings. We choose dragonfly as our subject because the four wings (two forewings and two hindwings) are not overlapping during flight. According to Noberg's observation [15] the flapping frequency of dragonfly (Aeschna juncea) is in the order of $27 \mathrm{~Hz}$.

\section{Base-excitation theory within the framework of a continuous system}

Consider an anisotropic inhomogeneous viscoelastic solid occupying a finite domain $R$ and bounded by the surface $\partial R$. The elastic constants $c_{i j k l}$ and mass density $\rho$ are functions of position vector $x_{i}$. The dynamic behavior of this solid can be described by the equation

$$
L u_{i}+C u_{i, t}=\rho u_{i, t t},
$$


where the absolute displacement field $u_{i}$ is a function of position vector and time $t$. The stiffness operator and damping operator are defined as

$$
\begin{gathered}
L u_{i} \equiv\left(c_{i j k l} u_{l, k}\right)_{, j}, \\
C \equiv \alpha L,
\end{gathered}
$$

where the standard subscript notation for partial derivative is used and summation convention is assumed. $\alpha$ is a constant. The boundary conditions of the solid are

$$
\begin{gathered}
u_{i}=\hat{u}_{i}\left(x_{j}, t\right) \text { on } \partial R_{u}, \\
T u_{i}=\hat{t}_{i}\left(x_{j}, t\right) \text { on } \partial R_{t},
\end{gathered}
$$

where the traction operator is defined as

$$
T u_{i} \equiv n_{j} c_{i j k l} u_{l, k}
$$

$\partial R_{u}$ and $\partial R_{t}$ are two disjoint subsets of the boundary $\partial R . \hat{u}_{i}$ and $\hat{t}_{i}$ are prescribed displacement and traction on the boundary. $n_{j}$ is a unit normal vector on $\partial R_{t}$. If the solid is attached to a rigid base which is driven translationally, the boundary conditions (4) and (5) become

$$
\begin{gathered}
u_{i}=d_{i}(t) \text { on } \partial R_{u}, \\
T u_{i}=0 \text { on } \partial R_{t},
\end{gathered}
$$

where $d_{i}$ is the displacement of the driven base.

In order to obtain the solution $u_{i}$ in Eq. (1) and inhomogeneous boundary conditions (7) and (8), eigenfunction expansion method is employed. It is well known that the normalized eigenfunctions $\Phi_{i}^{(r)}$ of the undamped hyperelastic solid subject to homogeneous boundary conditions are orthogonal. Mathematically, these orthogonal relations can be written in volume integrals as follows:

$$
\begin{gathered}
\int_{R} \Phi_{i}^{(r)} \Phi_{i}^{(s)} \mathrm{d} v=\delta_{r s}, \\
\int_{R} \Phi_{i}^{(r)} L \Phi_{i}^{(s)} \mathrm{d} v=-\omega_{r}^{2} \delta_{r s} .
\end{gathered}
$$

$\delta_{r s}$ is the Kronecker delta symbol and $\omega_{r}$ is the $r$ th undamped natural frequency. We express the solution $u_{i}$ of the solid under base excitation as

$$
u_{i}\left(x_{j}, t\right)=U_{i}\left(x_{j}, t\right)+d_{i}(t),
$$

where $U_{i}\left(x_{j}, t\right)$ is the displacement of the structure relative to the vibrating rigid base. Substituting Eq. (11) into Eq. (1) results in

$$
L U_{i}+C U_{i, t}=\rho U_{i, t t}+\rho \ddot{d}_{i}-C \dot{d}_{i},
$$

where $U_{i}$ satisfies the homogeneous boundary conditions

$$
\begin{gathered}
U_{i}=0 \quad \text { on } \partial R_{u}, \\
T U_{i}=0 \quad \text { on } \partial R_{t} .
\end{gathered}
$$

In order to solve this inhomogeneous boundary value problem, we express $U_{i}$ in terms of eigenfunctions of the undamped solid,

$$
U_{i}\left(x_{j}, t\right)=\sum_{r} q_{r}(t) \Phi_{i}^{(r)}\left(x_{j}\right)
$$

where $q_{r}(t)$ is the generalized coordinate. Substituting Eq. (15) into Eq. (12), with use of the orthogonality relations (9) and (10), and taking Fourier transform of the resulted equation, we can solve for $\tilde{q}_{r}(\omega)$. Consequently, the Fourier transform of the displacement field of the solid under base excitation can be 
derived as

$$
\tilde{u}_{i}\left(x_{j}, \omega\right)=\sum_{r}\left[\frac{\omega^{2} \int_{R} \rho \Phi_{j}^{(r)} \tilde{d}_{j} \mathrm{~d} v}{\omega_{r}^{2}+\mathrm{i} 2 \zeta_{r} \omega_{r} \omega-\omega^{2}} \Phi_{i}^{(r)}\right]+\tilde{d}_{i}(\omega),
$$

where $\mathrm{i}=\sqrt{-1}$ and the damping ratio is defined as

$$
\zeta_{r}=\frac{\alpha}{2 \omega_{r}} .
$$

In our experiment the rigid base is driven translationally in the $x_{1}$-direction. The measured input is the displacement of the base $\tilde{d}_{1}(\omega)$, and the output is the absolute displacement of the structure $\tilde{u}_{m}(\omega)$ at position $x_{j}$, respectively. Eq. (16) can be rewritten in the form

$$
\frac{\tilde{u}_{m}\left(x_{j}, \omega\right)}{\tilde{d}_{1}}=1+\sum_{r}\left(\frac{\omega^{2} B_{r} \Phi_{m}^{(r)}}{\omega_{r}^{2}+\mathrm{i} 2 \zeta_{r} \omega_{r} \omega-\omega^{2}}\right),
$$

where $\Phi_{m}^{(r)}$ is the $m$ th component of the $r$ th mode shape corresponding to the undamped natural frequency $\omega_{r}$. $B_{r}$ is a constant defined as

$$
B_{r}=\int_{R} \rho \Phi_{1}^{(r)} \tilde{d}_{1} \mathrm{~d} v
$$

The left-hand side of Eq. (18) represents the measured frequency response function in the experiment. The second term on the right-hand side is in the form compatible with the standard general purpose modal analysis software. Therefore, the measured frequency response function should be subtracted by a constant one before it can be fed into a commercial curve-fitting package to extract the modal parameters.

\section{Experimental set-up}

Fig. 1 shows the schematic diagram of the experimental apparatus we used in the base-excitation modal testing technique. The insect wing is attached to the vibrating base of an electro-mechanical shaker. An amplifier is responsible for providing sufficient power to the shaker to execute harmonic motion at certain frequency. When the base is moving harmonically the structure vibrates accordingly. A two-channel photonic probe (MTI 2000) serves as a non-contact sensor in measuring the vibration displacements. One of the probes

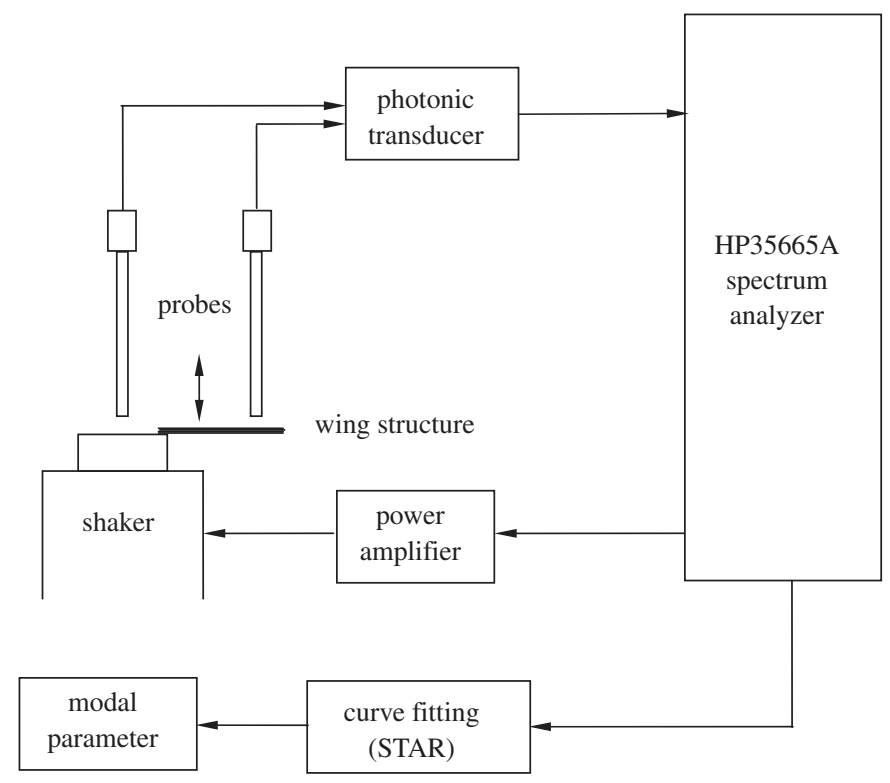

Fig. 1. Experimental set-up. 
measures the motion of the base (input signal), while the other measures the vibration at various locations of the structure (output signal). The number of measuring points on the structure depends on the size of the structure and the number of modes we are interested in. For a dragonfly wing we make the measurement at 25 locations. At each location these two signals (input and output) are fed into an HP 35665A spectrum analyzer simultaneously. The spectrum analyzer is capable of calculating the frequency response function. The spectrum analyzer facilitates a mode of operation called "swept-sine," which can vary the excitation frequency almost continuously between two specified frequencies. The frequency response function can be transported to and stored in a personal computer. After completing the testing at all designated points on the wing, the stored frequency response functions are fed into the modal testing software STAR, which is capable of performing curve-fitting and estimating the mode shapes of the structure and the associated damping.

The experimental technique described above is particularly suitable for ultra-light miniature structures for several reasons. First of all, the non-contact optical sensors will perform the measurement in a non-intrusive way. Secondly, this technique eliminates the need to apply and measure the point force on designated location of the structure, which becomes impractical when the structure is small. However, there is one drawback with this technique. The optical probe works well only when the surface of the structure is capable of reflecting back the light beam shone from the probe tip. The wing membrane is partly transparent and is not reflecting enough light back. Therefore, we have to put on small amount of silver paint on the spots of measurement. These silver paint spots will add additional mass on the insect wing, which inevitably change the natural frequencies. Therefore, the extracted natural frequencies should be interpreted with caution.

\section{Experimental procedure}

Two species of dragonflies (Orthetrum pruinosum and Orthetrum sabina) can be easily found in the countryside near Taipei during the spring and summer seasons. In the following we describe our experimental procedure on one of the wings. The same procedure has been repeated on 20 others. First of all the species of the dragonfly was identified as $O$. sabina. The whole dragonfly was weighed to be $0.130 \mathrm{~g}$. The left forewing was then severed from the thorax and was photographed as shown in Fig. 2(a). It is noted that the wing is far from being flat. The corner near the trailing edge close to the thorax curls upward for about $2 \mathrm{~mm}$. Therefore, it is more like a shell structure than a plate. The leading edge is much stiffer than the trailing edge. The veining
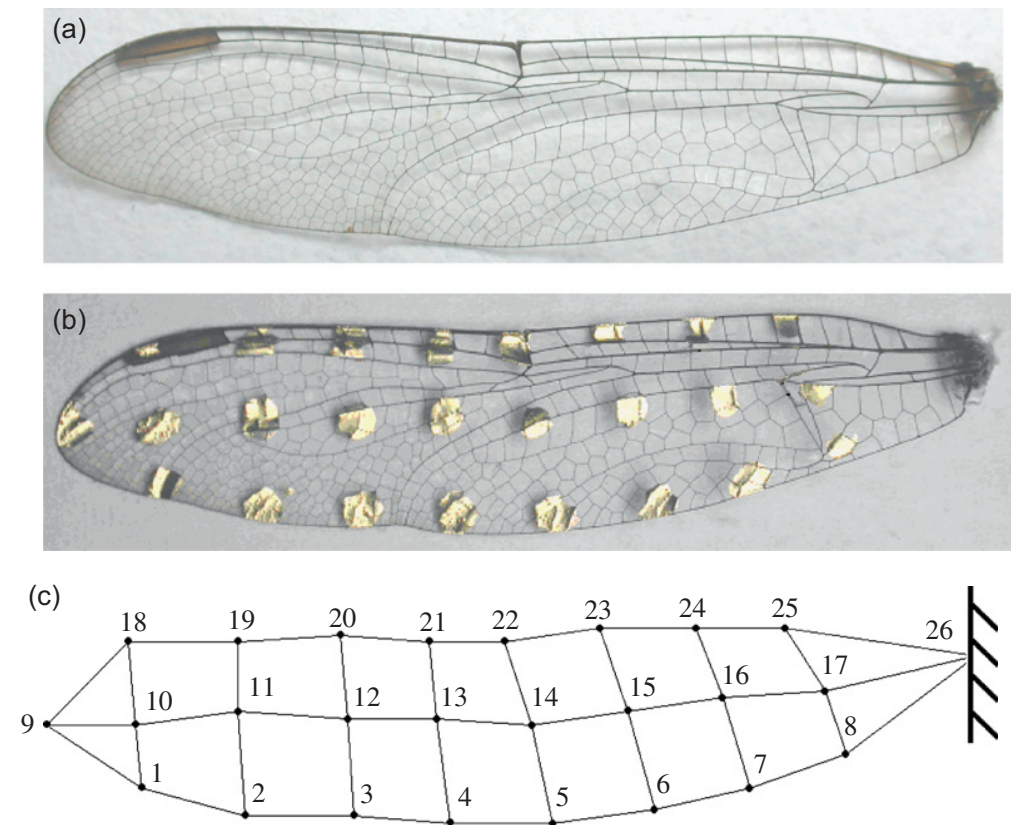

Fig. 2. (a) A forewing severed from the thorax; (b) the wing with 25 silver paint spots; and (c) the numbering system of the measured spots. 
on the wing forms many meshes on the surface, about 30 meshes in the longitudinal direction and about 15 meshes in the direction across from the leading edge to the trailing edge. These vein meshes serve as strong structure which is capable of resisting deformation during flight. Each mesh is covered with transparent membrane, which is to block the air from passing through the wing surface during flight.

The wing was weighed by a micro-scale to be $2.65 \mathrm{mg}$. The wing span from tip to base was measured as $38 \mathrm{~mm}$, while the maximum distance between the leading edge and the trailing edge was $8 \mathrm{~mm}$. We then put silver paint on 25 locations of the wing, as shown in Fig. 2(b). Each of these 25 locations was assigned a unique number, as shown in Fig. 2(c). Points 1-8 are near the trailing edge. Points 18-25 are near the leading edge. Point 9 is near the wing tip. The extra point labeled with number 26 represents the wing base and will be glued onto the vibrating base of the shaker. It is noted that point 26 is not painted. The boundary condition at base point 26 is close to the clamped condition in structural mechanics. The wing mass with silver paints on 25 points is weighed to be $3.42 \mathrm{mg}$. In other words, the silver paint increases the wing mass by about $30 \%$.

The experiment was conducted on a vibration isolation table to reduce contaminating noise from the floor. After gluing the wing onto the shaker base with cyanoacrylate-based adhesive we adjusted and calibrated the photonic probes so that one light beam was perpendicular to the shaker base and the other was perpendicular to one of the painted spots. After making certain that there was no resonance peaks below $100 \mathrm{~Hz}$ we specified the frequency of interest from 100 to $500 \mathrm{~Hz}$ for maximum accuracy. We initiated the swept-sine mode on the spectrum analyzer and started to measure the frequency response function. We repeated the same procedure for all 25 painted spots and transported the frequency response functions to a personal computer. The whole experimental procedure was completed within $24 \mathrm{~h}$ after the dragonfly was anesthetized and the wing was severed from the thorax. Figs. 3(a)-(c) are three sample frequency response functions for points 11, 14, and 7, respectively. In Fig. 3(a) we can observe two obvious peaks at 152 and 336 Hz. In Fig. 3(b) there are three peaks at 152, 284, and $340 \mathrm{~Hz}$. In Fig. 3(c) there are four obvious peaks at 152, 280, 340, and $404 \mathrm{~Hz}$. In the

(a)

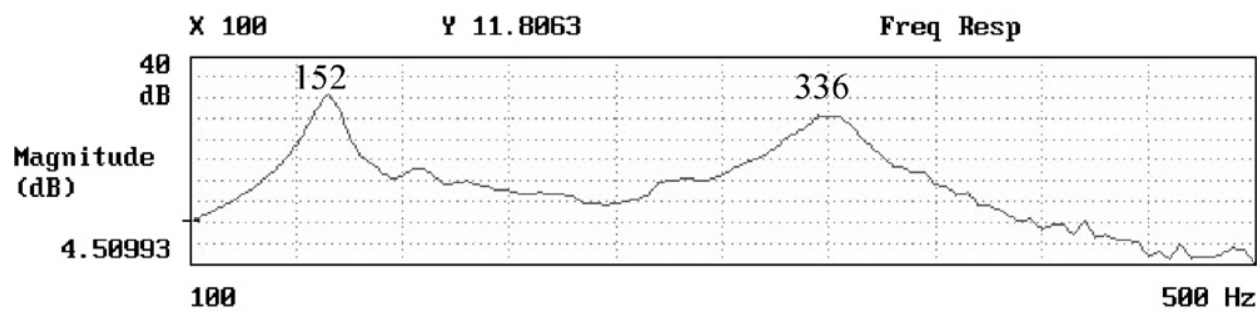

(b)

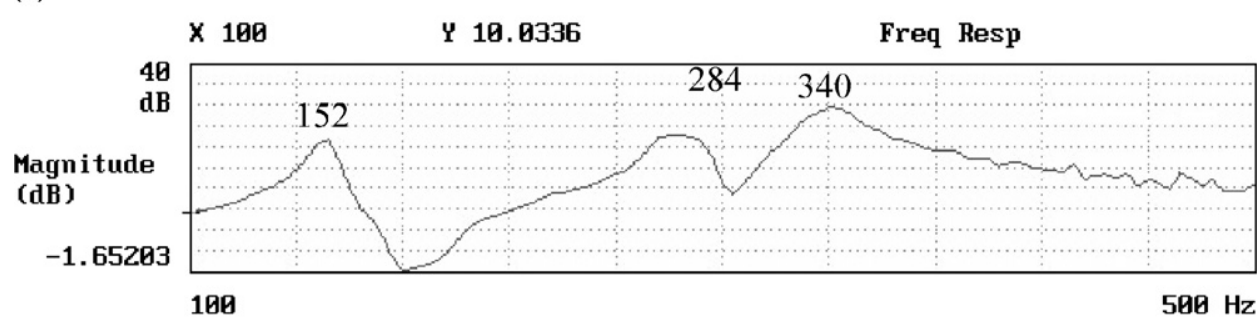

(c)

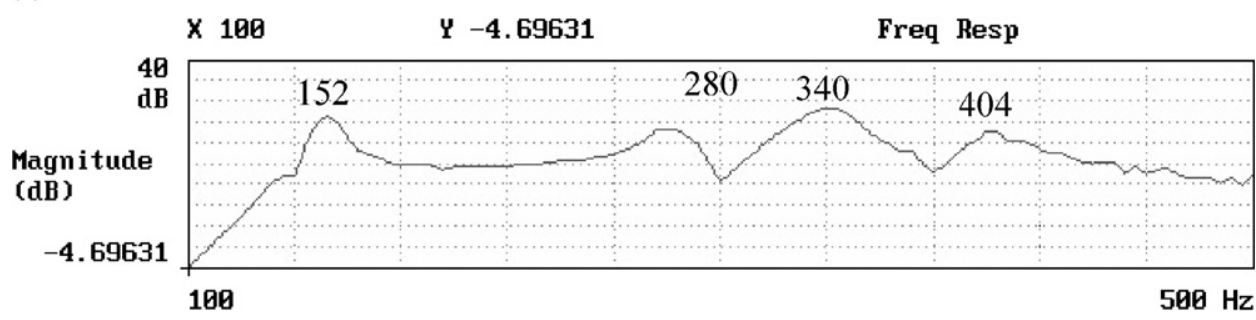

Fig. 3. Frequency response functions of points (a) 11, (b) 14, and (c) 7. 


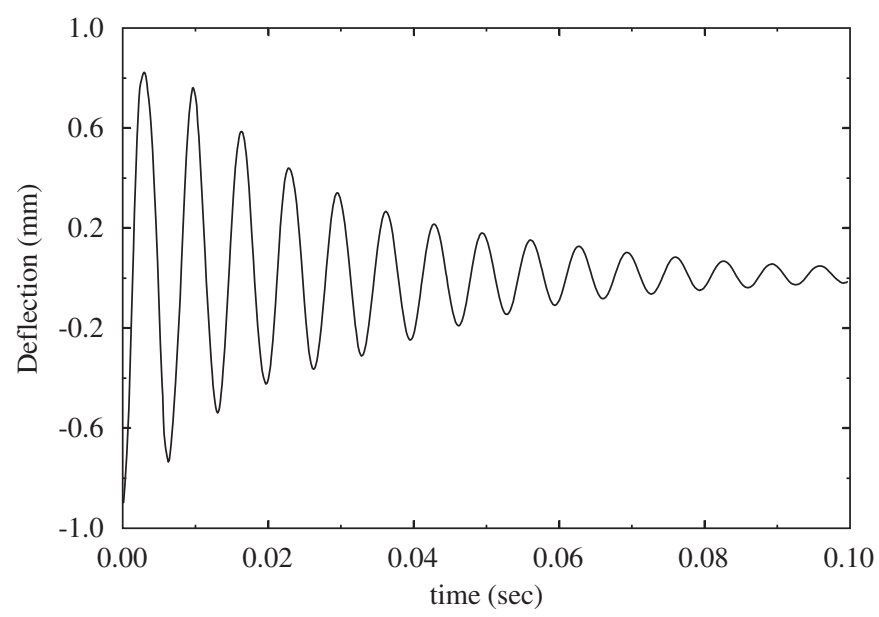

Fig. 4. Time history at point 9 after the wing is released from an initial deflection.

frequency response functions of other points, we may observe either two, three or four peaks, just like the ones in Fig. 3. After inspecting the 25 frequency response functions we concluded that for this particular specimen there are four natural modes between 100 and $500 \mathrm{~Hz}$. The averaged natural frequencies are identified as 150 , 289,337 , and $382 \mathrm{~Hz}$.

At this stage we conducted another experiment by deflecting the wing laterally a small displacement and releasing it. The displacement history at point 9 (the tip) was recorded in Fig. 4. From the oscillating displacement history we counted the number of peaks and verified the natural frequency as $151 \mathrm{~Hz}$. This confirmed the lowest natural frequency indicated by the frequency response function measurement. We can also determine the damping ratio as $4.2 \%$ by measuring the decaying rate of the consecutive peaks in Fig. 4 .

It is noted that the true natural frequency of the wing is the one without the silver paint. If we assume that the silver paint only adds mass onto the wing structure without changing its stiffness, then the true fundamental natural frequency (say, $\omega_{n}$ ) should be about $15 \%$ higher than the one measured experimentally $\left(\right.$ say, $\left.\omega_{p}\right)$. This can be seen from the relation

$$
\frac{\omega_{n}}{\omega_{p}}=\sqrt{\frac{m_{p}}{m_{n}}},
$$

where $m_{p}$ and $m_{n}$ are the wing masses with and without paint, respectively. In other words, the true fundamental natural frequency of this specimen is about $174 \mathrm{~Hz}$.

Our next step is to find the mode shapes corresponding to the four natural frequencies below $500 \mathrm{~Hz}$. We fed the 25 frequency response functions into the curve-fitting software. The software can extract the four mode shapes corresponding to the four natural frequencies, which are shown in Figs. 5(a)-(d). The original mesh is depicted with light lines in the background. Several comments regarding the mode shapes of this specimen can be summarized in the following: (1) first of all, as can be expected, the deformation of the leading edge is relatively small compared to the trailing edge in almost every mode. (2) Secondly, each mode shape contains both bending component and twisting component. In the first mode (Fig. 5(a)) we notice that the tip as well as part of the trailing edge near the tip has larger deformation compared to other parts. This indicates that the fundamental bending deformation dominates the first mode. (3) In the second mode (Fig. 5(b)) the tip and the leading edge are nearly fixed while the trailing edge has one obvious nodal point. This indicates that the higher-order bending deformation dominates this mode. (4) In the third mode (Fig. 5(c)) we can see obvious twisting deformation near the tip by observing that the two points near the tip, one on the leading edge and the other on the trailing edge, are moving in opposite directions. (5) The fourth mode (Fig. 5(d)) exhibits even higher order of bending deformation as we can observe two obvious nodal points on the trailing edge.

The mode shapes extracted by the modal testing technique can be real or complex. Real mode shapes mean that every point in the structure moves either in-phase or out-of-phase. On the other hand, the phase 


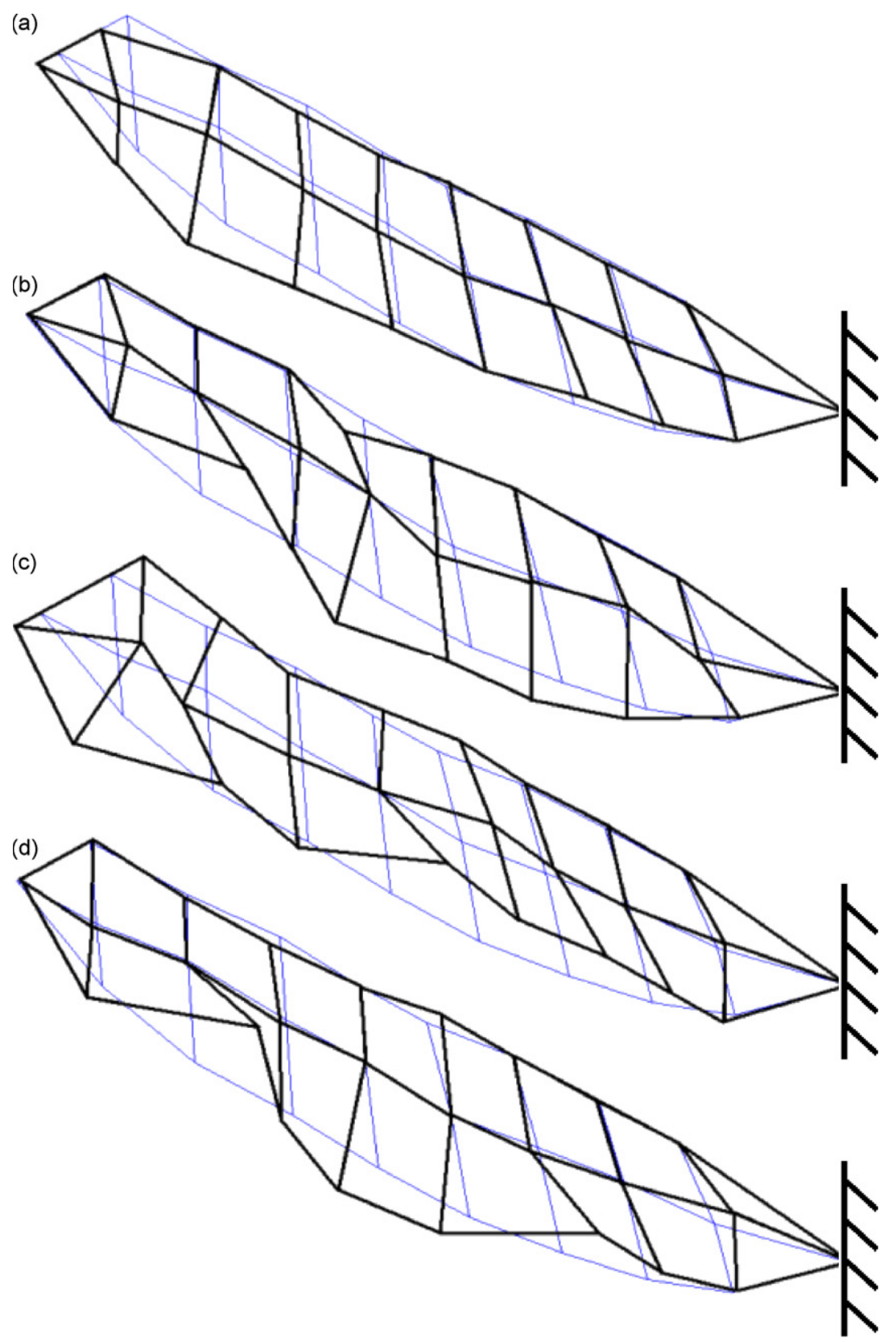

Fig. 5. (a)-(d) Mode shapes corresponding to the first four natural frequencies. (a) 150, (b) 289, (c) 337 and (d) $382 \mathrm{~Hz}$.

difference in points of a complex mode shape may be other than $0^{\circ}$ or $180^{\circ}$. As a consequence, for real mode shapes the nodal points are fixed in space. On the other hand, for complex modes the nodal points may move around within one period and the vibration looks like a wave propagation phenomenon. In the four modes depicted in Fig. 5, modes 1, 2, and 3 are close to real modes, while mode 4 is obviously a complex mode.

The curve-fitting software is also capable of estimating the damping ratio corresponding to each mode by inspecting the shape of the peaks in the frequency response function. The software assumes that the damping is of the viscous type, and estimates the damping ratios of modes $1-4 \mathrm{in} \mathrm{Fig.} 5$ to be $4.1 \%, 4.0 \%, 3.8 \%$, and $1.0 \%$, respectively. These values are not far from what we have estimated with the logarithmic decrement technique as explained in Fig. 4. 


\section{Discussions}

\subsection{Natural frequencies}

The experimental procedure described above was repeated for 20 other specimens. We ran the tests on wings from two species of dragonflies (Orthetrum pruinosum (OP) and Orthetrum sabina (OS)). The wing spans of the tested specimens range from 35 to $44 \mathrm{~mm}$, with an average about $39 \mathrm{~mm}$. Fig. 6 shows the recorded natural frequencies of 21 specimens. Four symbols are used in the figure; they are: $(\bigcirc)$ OS forewings; ( $(\bullet)$ OS hindwings $(\square)$ OP forewings; and ( $\square$ ) OP hindwings. We detected no obvious difference between the two different species, neither between the left and right wings. However, we do observe certain pattern difference between the fore- and hindwings, as will be explained later.

Fist of all, in the frequency range below $500 \mathrm{~Hz}$, we can detect three, four, or sometimes even five modes. These tests show that the measured fundamental natural frequency is in the range of $140-180 \mathrm{~Hz}$, with the average in the order of $150 \mathrm{~Hz}$. The average standard deviation of these 21 tests is $15 \%$. It is noted that these frequencies are for the wings with silver paint. By taking into account the added mass effect, we may conclude that the fundamental natural frequency of dragonflies is in the order of $170 \mathrm{~Hz}$.

To clarify the role of the inertial force on the wing deformation during insect flight, we simplify the wing structure into a simple mass-spring system under harmonic external force $F \sin \omega t$, where $F$ is the magnitude of the external force. This mass-spring system may represent the fundamental vibration mode of the dragonfly wing. The ratio between dynamic deformation amplitude $X_{d}$ and static deformation $X_{s}$, also called magnification factor, is [7]

$$
\frac{X_{d}}{X_{s}}=\frac{1}{1-\left(\omega / \omega_{n}\right)^{2}},
$$

where $\omega_{n}$ is the natural frequency of the mass-spring system, also the fundamental natural frequency of the dragonfly wing. The average flapping (excitation) frequency $\omega$ of a dragonfly is about $27 \mathrm{~Hz}$. Therefore, the excitation frequency is about $16 \%$ of the fundamental natural frequency $(170 \mathrm{~Hz})$. By using Eq. (21) the magnification factor is estimated to be 1.03 at this frequency ratio. In other words, taking into account the wing inertia only increases the deformation amplitude by $3 \%$ compared to static analysis ignoring wing inertia. Therefore, we conclude that inertial force of the wing is negligible compared to the elastic force during flapping flight. In other words, the wing deformation observed during insect flight is solely due to the balance between the external aerodynamic force and the elastic force of the wing structure. It is noted that this observation is contrary to the conjecture made in Ref. [6].

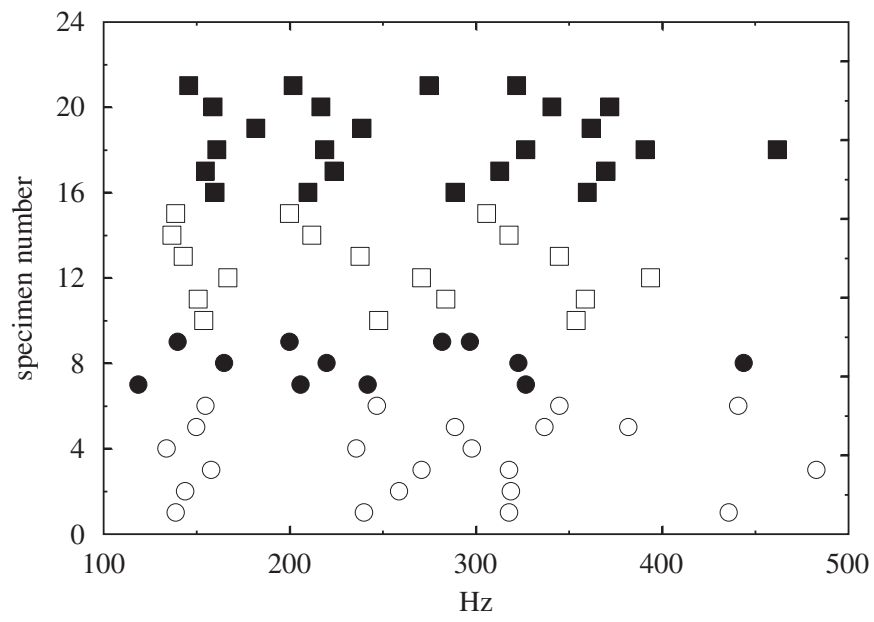

Fig. 6. Measured natural frequencies of 21 sample wings: (○) OS forewings; (๑) OS hindwings; ( $\square$ ) OP forewings and ( $\mathbf{\square})$ OP hindwings. 


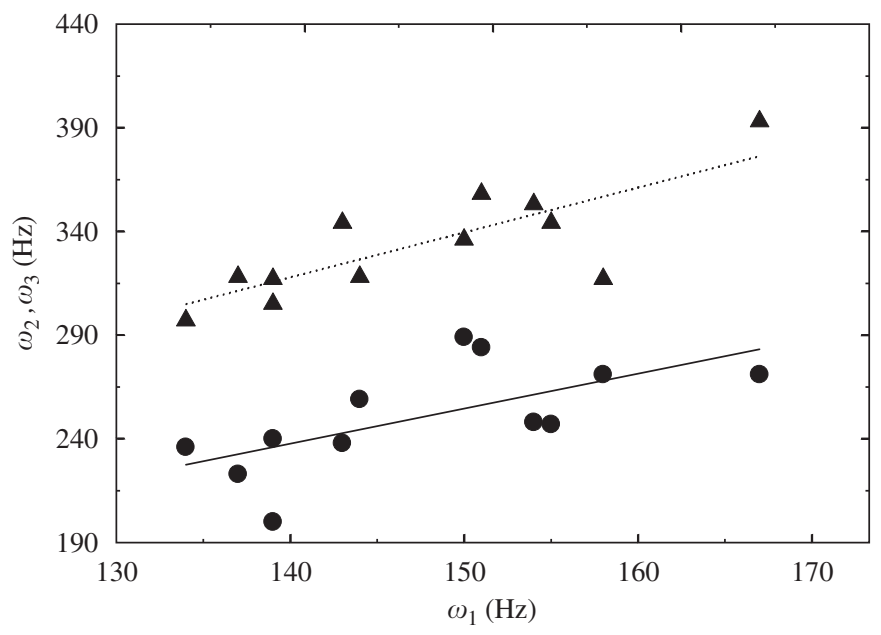

Fig. 7. The second natural frequency $\omega_{2}(\boldsymbol{\bullet})$ and the third natural frequency $\omega_{3}(\mathbf{\Delta})$ as functions of the fundamental frequency $\omega_{1}$ for the forewings.

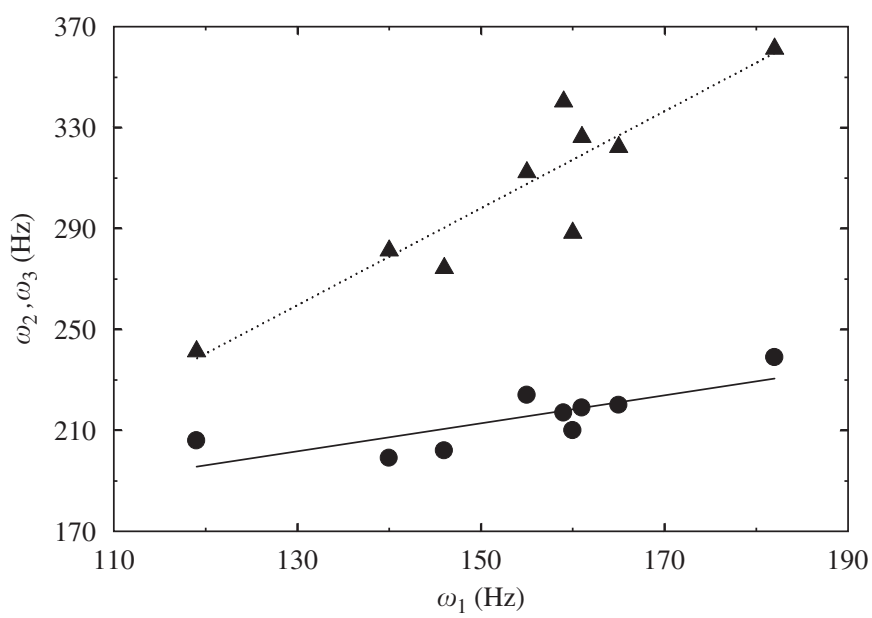

Fig. 8. The second natural frequency $\omega_{2}(\mathbf{\bullet})$ and the third natural frequency $\omega_{3}(\mathbf{\Delta})$ as functions of the fundamental frequency $\omega_{1}$ for the hindwings.

The natural frequencies of the higher modes scatter even more wildly than the fundamental mode. However, if we calculate the ratio of the second to the first and the ratio of the third to the first frequency, we can observe a certain pattern difference between the forewings and the hindwings. Figs. 7 and 8 show the relation between the second natural frequency $\omega_{2}(\boldsymbol{O})$ and the third natural frequency $\omega_{3}(\mathbf{\Delta})$ as functions of the fundamental frequency $\omega_{1}$ for the forewings (Fig. 7) and hindwings (Fig. 8), respectively. For the forewings we observe that the ratio between the first three natural frequencies is about 1:1.7:2.3. On the other hand, for the hindwings, the ratio between the first three natural frequencies is about 1:1.4:2.0.

The obvious pattern difference in the frequency ratio among the forewings and the hindwings prompts us to seek the source of this trend. One possible source is the geometric difference, especially the slenderness, between forewings and hindwings. In Fig. 9 we record the measured maximum widths and wing spans of the specimens we have tested. The symbols $(\mathbf{O})$ and $(\mathbf{A})$ are for the forewings and hindwings, respectively. Obviously, the forewings are in general slenderer than the hindwings. In order to characterize the slenderness of the wings while taking into account the shape difference, we adopt the definition of aspect ratio as the ratio 


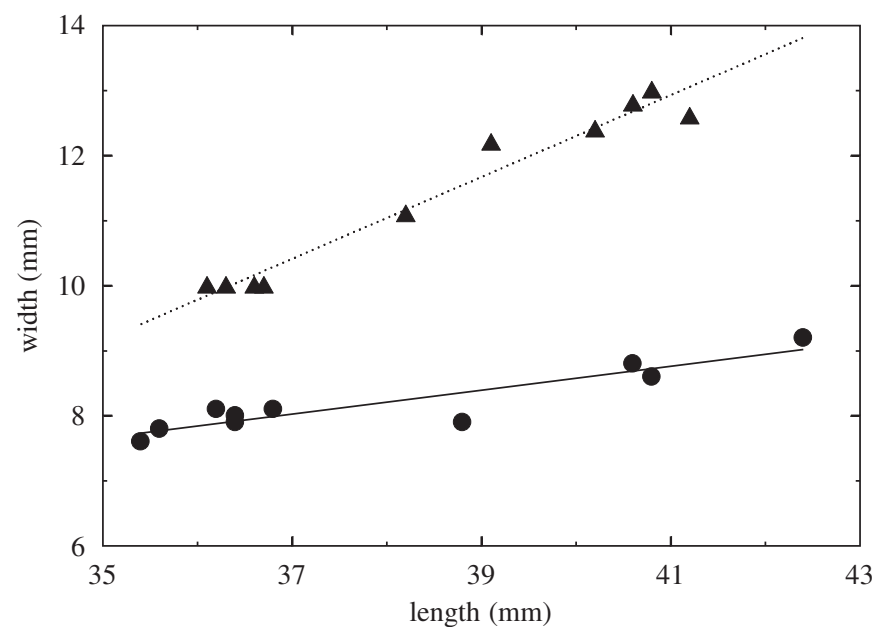

Fig. 9. The relation between the maximum width and span length of the forewings $(\boldsymbol{O})$ and the hindwings $(\mathbf{\Lambda})$.

between the square of the wing span and the wing area. According to our measurements and calculations, the average aspect ratios of the forewings and the hindwings are 5.37 and 4.39 , respectively. It is believed that the difference in the aspect ratio is responsible for the frequency pattern difference observed between the forewings and the hindwings.

\subsection{Mode shapes}

As have been explained in the preceding section, the mode shapes of the dragonfly wings can be considered as the combination of bending and twisting deformations. Although the mode shapes observed in Fig. 5 (for a forewing) are typical, there exist variations in some other specimens. For instance, we do observe twistingdominant deformation in the first mode of some specimens. The chance of this to occur is about one out of 10 specimens. Furthermore, we do not observe obvious difference between the forewings and the hindwings.

For the higher modes, the variation in mode shapes runs even more wildly. In the example shown in Fig. 5, three of the four modes are bending dominant, and one of them shows twisting-dominant deformation. However, in other tests we also saw specimens with three of them being twisting dominant and only one of them bending dominant. We are unable to make consistent conclusion regarding what these higher-order mode shapes should look like, except some of them are bending dominant and some of them are twisting dominant. Several possible reasons contribute to this variation. First of all we have to recognize the fact that although all dragonfly wings appear roughly the same, each of them is quite unique. The slight variation in wing shape and mesh structures may affect greatly the resulting mode shapes. Secondly, our experimental technique is not perfect. The photonic sensors require smooth targeting surface, while the wing surfaces are inevitably rough. Although this fact should not affect the measurement of the natural frequencies, it may affect the extraction of the mode shapes.

\subsection{Damping ratios}

Insect wings are generally lightly damped, with damping ratio in the order less than $5 \%$ when the experiment is conducted in the air. The average damping ratio of 5\% comes from two sources, one is from the wing structure itself, and the other from the air. It is expected that the measured damping will be smaller if the experiment is conducted in a vacuum chamber. It is noted that a total damping of $5 \%$ is considered to be very small. Therefore, the effect of the surrounding air on damping is insignificant. In addition, since the insects fly in the air, it makes more sense in measuring the total damping as far as the dynamic response of the flapping wings is concerned. 


\section{Conclusions}

In this paper, we used a base-excitation modal testing technique to measure the natural frequencies, mode shapes, and the associated damping of dragonfly wings severed from thoraxes. One of the purposes of the test is to clarify the role of inertial force in the wing deformation during insect flight. Several conclusions can be summarized as follows:

(1) The fundamental natural frequency of dragonfly wings is estimated to be in the order of $170 \mathrm{~Hz}$. The flapping frequency $27 \mathrm{~Hz}$ of dragonflies is about $16 \%$ of the fundamental natural frequency. At this frequency ratio, the inertial force of the wing is negligible compared to the elastic force. In other words, the wing deformation during flapping flight is solely due to the balance between the external aerodynamic force and the elastic force of the wing structure.

(2) Each mode shape contains both bending and twisting components. It is often observed that bending deformation dominates the fundamental mode. This information, together with the observed natural frequency, may be useful in establishing a more precise finite element model for the wing structure in the effort to predict the aerodynamic force during insect flight.

(3) The wing structures are generally lightly damped, with damping ratio in the order less than $5 \%$.

\section{References}

[1] R.J. Wootton, The mechanical design of insect wings, Scientific American November (1990) 114-120.

[2] D.J.S. Newman, R.J. Wootton, An approach to the mechanics of pleating in dragonfly wings, Journal of Experimental Biology 125 (1986) 361-372.

[3] A.R. Ennos, The importance of torsion in the design of insect wings, Journal of Experimental Biology 140 (1988) $137-160$.

[4] R.J. Wootton, Leading edge section and asymmetric twisting in the wings of flying butterflies (Insecta, Papilionoidea), Journal of Experimental Biology 180 (1993) 105-117.

[5] S.A. Combes, T.L. Daniel, Flexural stiffness in insect wings I. Scaling and the influence of wing venation, Journal of Experimental Biology 206 (2003) 2979-2987.

[6] T.L. Daniel, S.A. Combes, Flexible wings and fins: bending by inertial or fluid-dynamic forces, Integrative Comparative Biology 42 (2002) 1044-1049.

[7] J.P. Den Hartog, Mechanical Vibrations, McGraw-Hill Book Company, Inc., New York, 1956.

[8] H.R. Radwan, J.V. Chokshi, Use of non-contact measurements for modal analysis of disk drive components, Proceedings of the Third IMAC, Orlando, FL, 1985, pp. 490-496.

[9] J. Castagna, A method of determining the modes of vibration of disc drive heads and suspensions during operation, Proceedings of the Sixth IMAC, Kissimmee, FL, 1988, pp. 745-750.

[10] M.E. Patton, M.W. Trethewey, A technique for nonintrusive modal analysis of ultralightweight structures, Proceedings of Fifth IMAC, London, England, 1987, pp. 1425-1431.

[11] C. Wilson, D.B. Bogy, Experimental modal analysis of a suspension assembly loaded on a rotating disk, ASME Journal of Vibration and Acoustics 116 (1994) 85-92.

[12] D.K. Miu, G.M. Frees, R.S. Gompertz, Tracking dynamics of read/write head suspensions in high-performance small form factor rigid disk drives, ASME Journal of Vibration and Acoustics 112 (1) (1990) 33-39.

[13] J.-G. Beliveau, F.R. Vigneron, Y. Soucy, S. Draisey, Modal parameter estimation from base excitation, Journal of Sound and Vibration 107 (3) (1986) 435-449.

[14] D. Henze, R. Karam, A. Jeans, Effects of constrained-layer damping on the dynamics of a type 4 in-line head suspension, IEEE Transactions on Magnetics 26 (5) (1990) 2439-2441.

[15] R.A. Norberg, Hovering flight of the dragonfly Aeschna juncea L.: kinematics and aerodynamics, in: T.Y. Wu, C.J. Brokaw, C. Brennen (Eds.), Swimming and Flying in Nature, Vol. 2, Prenum Press, New York, 1975, pp. 763-781. 